\title{
Basal buoyancy and fast-moving glaciers: in defense of analytic force balance
}

\author{
C. J. van der Veen \\ Department of Geography and Atmospheric Science, University of Kansas, 203 Lindley Hall, 1475 Jayhawk Blvd, Lawrence, \\ KS 66045, USA \\ Correspondence to: C. J. van der Veen (cjvdv@ku.edu)
}

Received: 11 February 2016 - Published in The Cryosphere Discuss.: 24 February 2016

Revised: 31 May 2016 - Accepted: 6 June 2016 - Published: 29 June 2016

\begin{abstract}
The geometric approach to force balance advocated by $\mathrm{T}$. Hughes in a series of publications has challenged the analytic approach by implying that the latter does not adequately account for basal buoyancy on ice streams, thereby neglecting the contribution to the gravitational driving force associated with this basal buoyancy. Application of the geometric approach to Byrd Glacier, Antarctica, yields physically unrealistic results, and it is argued that this is because of a key limiting assumption in the geometric approach. A more traditional analytic treatment of force balance shows that basal buoyancy does not affect the balance of forces on ice streams, except locally perhaps, through bridging effects.
\end{abstract}

\section{Introduction}

Ice streams are fast-moving rivers of ice embedded in the more sluggish-moving main body of ice sheets, and are responsible for the bulk of drainage from the interior in West Antarctica. Most ice streams start well upstream from the coast, some extending several hundreds of kilometers into the interior, and drain into floating ice shelves or ice tongues and are believed to represent the transition from inland-style "sheet flow" to ice-shelf spreading. The nature of this transition remains under debate, however.

In a long series of papers, T. Hughes presents the geometric approach to the balance of forces acting on ice shelves, ice streams, and interior ice (Hughes, 1986, 1992, 1998, 2003, 2009a, b, 2012; Hughes et al., 2011, 2016). Rather than working his way through the basic equations, as done by most other investigators, including Van der Veen and Whillans (1989) and Van der Veen (2013), he presents deriva- tions based on graphical interpretation of triangles representing forces acting on an ice column. In essence, the transition in flow regime is achieved by introducing a basal buoyancy factor that describes the gradual ice-bed decoupling towards the grounding line.

The idea of basal buoyancy has been invoked many times before in glaciology, in particular in the context of formulating a sliding relation. In many models, the sliding speed is assumed to be inversely proportional to the "effective basal pressure" defined as the difference between the weight of the overlying ice and the pressure in the subglacial drainage system. Intuitively, this approach may seem to make sense: as the subglacial water pressure increases, the normal force on the bed should be reduced, thus allowing the glacier to move faster. However, this does not affect the balance of forces in the horizontal direction, as suggested by Hughes (2008, 2012).

The objective of this brief note is to evaluate the implications of Hughes' geometric approach to force balance by applying the results to Byrd Glacier, East Antarctica.

\section{Force balance: analytic approach}

Analytic treatments of glacier force balance are numerous and derivations of the depth-integrated force-balance equations are now standard fare in most glaciology textbooks. In most cases, this balance of forces is discussed in terms of stress deviators, defined as the full stress minus the hydrostatic pressure. This is done because the flow law for glacier ice relates strain rates to stress deviators. That is

$\sigma_{i j}^{\prime}=\sigma_{i j}-\frac{1}{3} \delta_{i j}\left[\sigma_{x x}+\sigma_{y y}+\sigma_{z z}\right]$, 
where the prime denotes the stress deviator and unprimed stresses are full stresses, and $\delta_{i j}=1$ for $i=j$ and $\delta_{i j}=0$ for $i \neq j$. Deviatoric stresses are called for in the flow law for glacier ice because the rate of deformation is in good approximation independent of the hydrostatic pressure. However, the use of deviatoric stresses in discussing the balance of forces unnecessarily complicates the interpretation because the longitudinal deviatoric stress in one direction depends on the full normal stresses in all three directions of a Cartesian coordinate system. It is more convenient to consider stresses in a glacier as the sum of the stress due to the weight of the ice (lithostatic stress) and stresses, $R_{i j}$, due to the flow (resistive stresses). This partitioning makes a clearer distinction between action and reaction in glacier dynamics (Whillans, 1987) and follows common practice in geophysics (Engelder, 1993, p. 10; Turcotte and Schubert, 2002, p. 77).

It may be noted that the term "resistive" stress is an unfortunate choice, perhaps, because these stresses do not necessarily always offer resistance to flow. For example, gradients in longitudinal stress can act in cooperation with the driving stress in pulling the ice forward. The more appropriate terminology would perhaps be flow stress or, following geophysical terminology, tectonic stress. The $R_{i j}$ represents the stresses that are associated with glacier deformation, as opposed to the lithostatic stress which describes the action of gravity. However, the existing terminology appears to have made its way into the glaciological literature (e.g., Cuffey and Paterson, 2010, Sect. 2.2) and a name change at this stage likely would introduce even more confusion.

Van der Veen (2013, Sect. 3.1) presents a derivation of the column-average balance equations by integrating the momentum balance equations over the full ice thickness. Van der Veen and Payne (2004) and Van der Veen (2013, Sect. 3.2) present a discussion of force balance based on geometric arguments and, not surprisingly, arrive at the same result. Without loss of generality, flow in one horizontal direction may be considered. That is, the horizontal $x$ axis is chosen in the direction of flow and it is assumed that there is no component of flow in the other horizontal $y$ direction. The $z$ axis is vertical upward, with $z=0$ at sea level. Force balance in the flow direction is then described by the following equation (Van der Veen and Whillans, 1989; Van der Veen, 2013, Sect. 3.1):

$\tau_{d x}=\tau_{b x}-\frac{\partial}{\partial x}\left(H \widetilde{R}_{x x}\right)-\frac{\partial}{\partial y}\left(H \widetilde{R}_{x y}\right)$.

In this expression, $\tau_{d x}$ denotes the gravitational driving stress, defined as

$\tau_{d x}=-\rho g H \frac{\partial h}{\partial x}$,

where $\rho$ represents the density of ice, $g$ the gravitational acceleration, $H$ the ice thickness, and $h$ the elevation of the upper ice surface. The terms on the right-hand side of
Eq. (2) represent the resistance to flow associated with, respectively, drag at the glacier base, gradients in longitudinal stress ("pulling power"), and lateral drag arising from shear between the faster-moving ice stream and the near-stagnant interstream ridges or fjord walls. The tilde $(\sim)$ denotes depthaveraged values. Resistive stresses are defined following Van der Veen and Whillans (1989):

$R_{x x}=\sigma_{x x}+\rho g(h-z)$

$R_{x y}=\sigma_{x y}$,

where $\sigma_{i j}$ represents the full stress, and $-\rho g(h-z)$ the lithostatic stress (weight of the ice above) at depth $z$.

The balance Eq. (2) is exact. No approximations are involved in deriving this expression from the basic equations describing the balance of forces on a segment of ice (Van der Veen and Whillans, 1989; Van der Veen, 2013, Sect. 3.1). Consequently, this equation applies to freefloating ice shelves where the gravitational driving stress is balanced entirely by gradients in longitudinal stress, yielding the classic Weertman (1957) solution (Van der Veen, 2013, Sect. 4.5), as well as laminar flow with basal drag providing sole resistance to flow (Van der Veen, 2013, Sect. 4.2). Except for these two end-member solutions, Eq. (2) does not permit analytic solutions without making additional assumptions. Nevertheless, because no approximations were made in its derivation, balance Eq. (2) applies equally well to transitory flow regimes such as ice streams and outlet glaciers.

Integrating the balance equation over the width of the flow band simplifies the resistive term associated with drag at the lateral margins. Denoting the lateral shear stress at the margins by $\tau_{\mathrm{S}}$ (assumed to have the same magnitude but opposite signs at both lateral margins), and glacier width by $W$, lateral resistance on a section of glacier of unit width is (Van der Veen, 2013, Eq. 4.39)

$F_{\mathrm{s}}=\frac{2 H \tau_{\mathrm{s}}}{W}$

and the width-averaged force-balance equation becomes

$-\rho g H \frac{\partial h}{\partial x}=\tau_{b x}-\frac{\partial}{\partial x}\left(H \widetilde{R}_{x x}\right)+\frac{2 H \tau_{\mathrm{s}}}{W}$

with the understanding that all terms are averaged over the flow-band width (or, equivalently, considered constant across the flow band), and Eq. (3) has been substituted for the driving stress on the left-hand side. Note that contrary to what Hughes (2008, p. 53) states, lateral drag does not vanish at the center of a glacier. While the shear stress, $R_{x y}$, is zero at the centerline, its transverse derivative and thereby resistance from lateral drag, is not zero there. In fact, according to Eq. (6), this resistance is constant across the glacier width.

The geometric approach developed by Hughes arrives at a similar balance equation, namely

$$
-\rho g H \frac{\Delta h}{\Delta x}=\tau_{b}-\frac{\Delta H \sigma_{F}}{\Delta x}+\frac{2 H \tau_{\mathrm{s}}}{W}
$$


(Hughes, 2003, Eq. 36), or taking the limit $\Delta x \rightarrow 0$

$-\rho g H \frac{\partial h}{\partial x}=\tau_{b}-\frac{\partial H \sigma_{F}}{\partial x}+\frac{2 H \tau_{\mathrm{s}}}{W}$.

In these balance equations, $\sigma_{F}$ is related to the deviatoric tensile stress; its exact interpretation has evolved over the years. To avoid unnecessary confusion, a consistent notation is used in the following discussion, based on Hughes $(2008,2012)$. Comparison of Eqs. (7) and (9) shows that $\sigma_{F}=\widetilde{R}_{x x}$. It is the way this stress is calculated that sets Hughes' geometric approach apart from the analytic approach. In essence, this stress is linked to basal buoyancy and, in later versions, downglacier-integrated resistance from basal and lateral drag. While the force-balance Eq. (7) does not imply any assumption about the depth-variation in the longitudinal resistive stress, $R_{x x}$, Hughes (2003) explicitly argues that both $\sigma_{F}$ and the associated stretching rate, $\dot{\varepsilon}_{x x}$, must be constant in the vertical direction.

\section{Force balance: geometric approach}

Discussing force balance for stream flow, Hughes (2008, Sect. 11) equates $\sigma_{F}$ with a basal buoyancy factor, $\varphi$, as

$\sigma_{F}=\frac{\rho g H}{2} \phi^{2}$,

where

$\phi=\frac{\rho_{\mathrm{w}} H_{\mathrm{w}}}{\rho H}=\frac{P_{\mathrm{w}}}{P_{i}}$

is determined by the ratio of the areal average water pressure under the ice, and basal ice pressure (or weight of the ice column); $\rho_{\mathrm{w}}$ represents the density of sea water. For a floating ice shelf, $\varphi=1$, and Eq. (10) reduces to the solution for a free-floating ice shelf spreading in the $x$ direction only (Weertman, 1957; Van der Veen, 2013, Sect. 4.5). For inland-style flow, $\varphi=0$, and the lamellar flow solution can be derived. For ice streams and outlet glaciers that represent the transition from interior-style flow to ice-shelf spreading, $0<\varphi<1$. In first-order approximation

$\phi=\frac{H_{o}}{H(x)}$,

where $H_{o}$ represents the thickness at the grounding line, and $H(x)$ the ice thickness at some distance $x$ upstream of the grounding line (Hughes, 2008, Eq. 11.11). This relation is robust and a decrease in $\varphi$ going upglacier from the grounding line increases ice-bed coupling and generally yields a concave surface profile (Hughes, 2008, p. 58).

Hughes (2008) takes the geometric approach to another level and relates all resistance to flow on ice streams to the basal buoyancy factor, $\varphi$. In addition to relating the longitudinal stress deviator to this factor, lateral and basal drags are linked to $\varphi$ as (Hughes, 2008, Table 12.1; see also Hughes, 2009a, b; Hughes, 2012, Table 12.1; Hughes et al., 2016; Eqs. 12-17)

$$
\begin{aligned}
& \tau_{b}=-\rho g H(1-\phi)^{2} \frac{\partial h}{\partial x}-\rho g H^{2}(1-\phi) \frac{\partial \phi}{\partial x} \\
& F_{\mathrm{s}}=\frac{2 H \tau_{\mathrm{s}}}{W}=-2 \rho g H \phi(1-\phi) \frac{\partial h}{\partial x} \\
& -\frac{1}{2} \rho g H W(1-2 \phi) \frac{\partial \phi}{\partial x},
\end{aligned}
$$

while the longitudinal stress gradient term is given by

$$
\frac{\partial H \sigma_{F}}{\partial x}=\rho g H \phi\left(\phi \frac{\partial h}{\partial x}+H \frac{\partial \phi}{\partial x}\right) \text {. }
$$

These equations are derived without consideration of ice velocity or physical properties of the ice (temperature, stiffness, fabric development, etc.) or, for that matter, basal water availability and balance. Presumably, all these factors are somehow reflected in the ice-stream geometry and the inferred basal buoyancy.

\section{Geometric approach: application to Byrd Glacier, Antarctica}

Balance of forces on Byrd Glacier, East Antarctica, was first discussed by Whillans et al. (1989), who used measurements of surface velocity and surface topography derived from repeat aerial photogrammetry, to evaluate the relative roles of lateral drag, gradients in longitudinal stress, and basal drag in resisting the gravitational driving stress. Van der Veen et al. (2014) reconsidered these calculations and also investigated the effect of drainage of two sub-glacial lakes in the catchment region. Both studies employed the analytic forcebalance approach.

Reusch and Hughes (2003), Hughes (2009a), and Hughes et al. $(2011,2016)$ discuss force balance on Byrd Glacier from the geometric perspective and take issue with the analytic approach of Whillans et al. (1989). None of these studies explicitly shows how the various resistive forces vary along the glacier and, instead, largely base their discussion on how the basal buoyancy, $\varphi$, varies upstream of the grounding line. Therefore, to fully appreciate the implications of the geometric approach, Eqs. (13)-(15) are applied here to evaluate all terms in the balance of forces.

The geometry is shown in Fig. 1 (Van der Veen et al., 2014, Fig. 6). Only the lower $30 \mathrm{~km}$ stretch upstream of the grounding line (at $x=-10 \mathrm{~km}$ ) is considered here because that is the region laterally bounded by near-parallel fjord walls. Also shown in Fig. 1 is the basal buoyancy factor calculated from Eq. (12); $\varphi$ increases from around 0.7 a little more than $30 \mathrm{~km}$ upstream of the grounding line, to 1 where the ice starts to float. While there is nothing in particular wrong or disturbing about this basal buoyancy factor, the situation becomes more problematic when the actual forces are considered. 


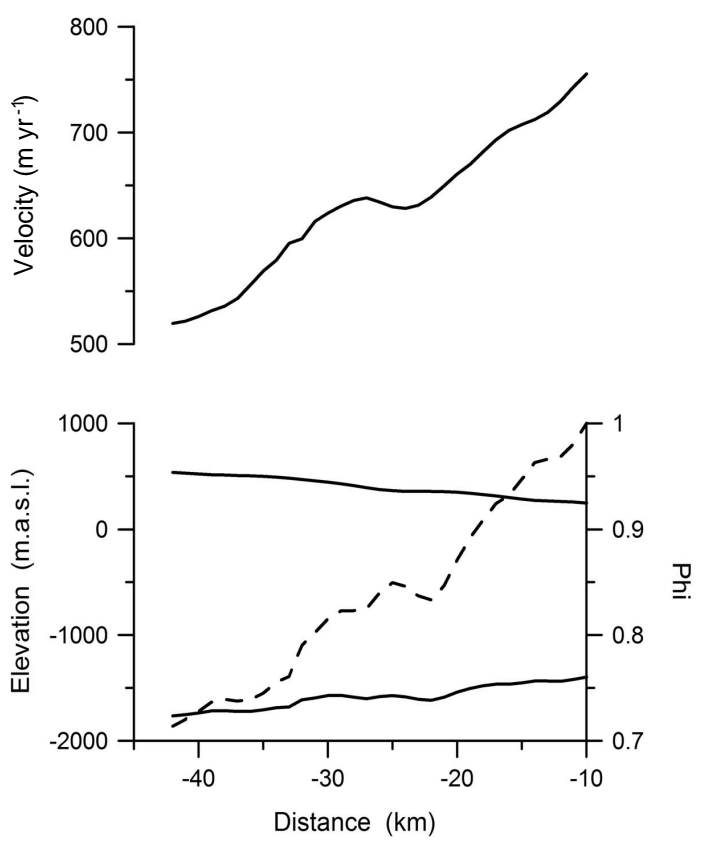

Figure 1. Geometry of the lower part of Byrd Glacier, East Antarctica. The dashed line in the lower panel shows the buoyancy factor, calculated from Eq. (12).

The average driving stress is $\sim 160 \mathrm{kPa}$, but shows large spatial variations that appear to be temporally fixed (Fig. 2). Gradients in longitudinal stress are mostly negative, averaging $-140 \mathrm{kPa}$ along the flow line, implying that, except in a few isolated locations, this term acts in the same directions as the driving stress, draining the grounded ice into the Ross Ice Shelf. To maintain balance of forces, flow resistance is partitioned between basal drag $(\sim 53 \mathrm{kPa})$ and lateral drag $(\sim 247 \mathrm{kPa})$. In the geometric approach, the bulk of flow resistance is associated with lateral drag and basal drag supports only about one-third of the driving stress. The finding that longitudinal stress gradients act in cooperation with the driving stress over a distance of more than $30 \mathrm{~km}$ is surprising, and there is no credible physical mechanism that can explain this. Even on a free-floating ice shelf, where other sources of flow resistance may be neglected, gradients in longitudinal stress arising from water pressure act to oppose the driving stress (Weertman, 1957; Van der Veen, 2013, Sect. 4.5). Hughes et al. (2016, p. 201) argue that the water buttressing produces a back stress in the longitudinal force balance, and that this is a real stress that is obscured using continuum mechanics in the conventional analytic approach. According to Hughes $(2008,2012)$, this stress, or "pulling power", results in the overestimation of longitudinal stress gradients, adding to the driving stress.
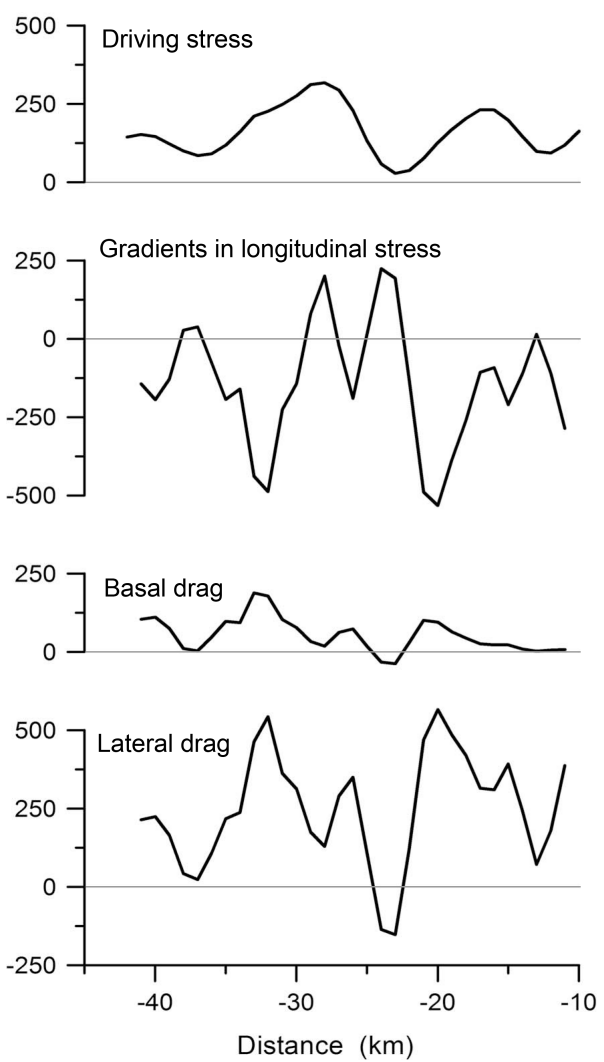

Figure 2. Force-balance terms on Byrd Glacier according to geometric force balance, Eqs. (13)-(14).

\section{Limitation of the geometric approach}

To understand the limitation in the geometric approach to force balance, consider the forces along an ice stream flow line as discussed in Hughes (2008, p. 53 ff.) (see also Fig. 1 in Hughes (2003), and Hughes, 2012; Sect. 11). The geometry is shown in Fig. 3. While Hughes (2008, p. 53; 2012, p. 66) erroneously states that resistance from lateral drag vanishes at the centerline of an ice stream and therefore does not include this source of resistance in his discussion, this has no significant impact on the following discussion - lateral drag can be readily added to the basal drag term without altering the general tenets of the analysis.

According to Hughes $(2008,2012)$, the gravitational driving force at $x$ is

$F_{\mathrm{g}}=\operatorname{areaADF}=\frac{1}{2} \rho g H^{2}$

and this force must be balanced by longitudinal resisting forces consisting of a "water buttressing force" (area CDE), a tensile force (area BCE), and a basal drag force (area ABEF). The basal drag force equals integrated basal resistance from the grounding line to the upglacier location (integrated resistance from lateral drag could also be included in this term). The area of each triangle is obtained from the familiar for- 


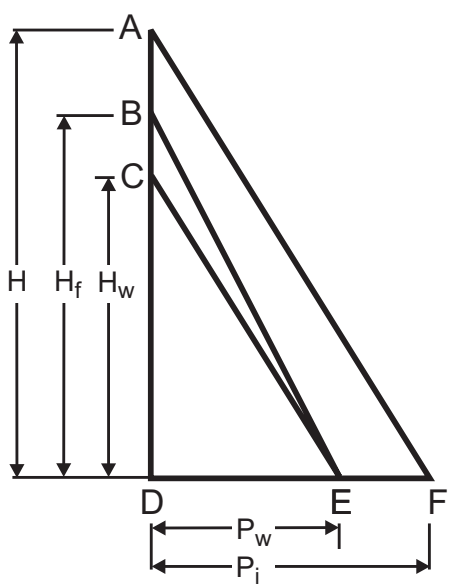

Figure 3. Geometric force balance according to Hughes (2008). $H$ represents ice thickness, $H_{\mathrm{f}}$ the flotation height or height of the ice column supported by basal water pressure, and $H_{\mathrm{W}}$ the piezometric height; $P_{\mathrm{W}}$ and $P_{i}$ represent the basal water pressure and weight of the ice column, respectively. Ice flow is from right to left.

mula (base $\times$ height) $/ 2$, where the base either equals the ice overburden pressure $(\mathrm{DF}=\rho \mathrm{gH})$ or water pressure $\left(\mathrm{DE}=\rho_{\mathrm{w}}\right.$ $\mathrm{gH})$, and the height equals the ice thickness $(\mathrm{AD}=H)$, flotation height $\left(\mathrm{BD}=H_{\mathrm{f}}=\left(\rho_{\mathrm{w}} / \rho\right) H_{\mathrm{w}}\right)$, or the piezometric height $\left(\mathrm{CD}=H_{\mathrm{w}}=P_{\mathrm{w}} /\left(\rho_{\mathrm{w}} g\right)\right)$. Thus, each of the resistive terms can be evaluated as a function of local ice thickness and water pressure. The reason why, for example, area $\mathrm{ABEF}$ should be associated with basal drag force (or basal plus lateral drag) remains unclear but is irrelevant.

The problem with this reasoning is that $F_{\mathrm{g}}$ does not represent the gravitational driving force. Rather, this force equals the lithostatic force associated with the weight of ice. When considering horizontal forces at any location, this force is balanced exactly by an equal but opposite force from ice of equal thickness on the left of the vertical line A-D, except at the calving front. In other words, adhering to the geometric representation, triangle A-D-F is balanced by the mirror triangle A-D-P (Fig. 4a), whether one considers an ice shelf, ice stream, or interior ice. The gravitational force that drives glacier flow is associated with gradients in lithostatic stress (Fig. 4b). A correct geometry-based discussion of force balance would consider the difference between lithostatic stress at $x$ and at some location $x+\Delta x$ downglacier, and, in the case of a sloping bed, lithostatic stress acting on the bed, and the difference between longitudinal stress at both locations, in addition to basal and lateral drag acting over the distance considered. Doing so gives the balance Eq. (7) with the term on the left-hand side representing the driving stress (Van der Veen and Payne, 2004; Van der Veen, 2013, Sect. 3.2).

It is not possible to relate resistive forces at any location to point values such as basal water pressure or weight of the ice at location $x$. While resistive stresses, such as $R_{x x}$, can be evaluated at specific points, resistance to flow is associ- (a)

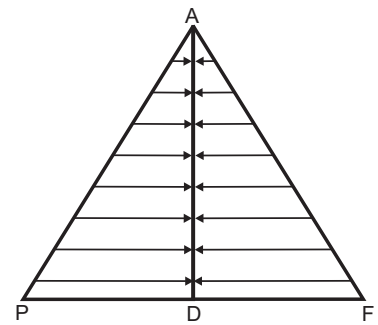

(b)

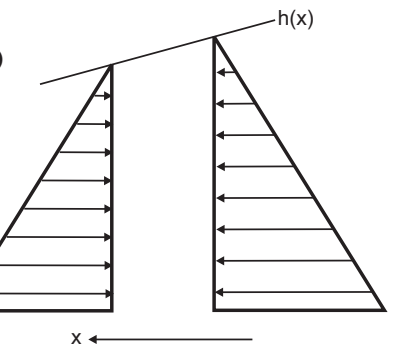

Figure 4. (a) At any location the lithostatic stress increases linearly with depth from zero at the ice surface to $\rho \mathrm{gH}$ at the base; the lithostatic stress from ice on the right of the vertical line A-D is balanced by an equal but opposite lithostatic stress from ice on the right and the area of triangle A-D-F equals that of triangle A-D-P. (b) Gradients in lithostatic stress are associated with a sloping ice surface, $h(x)$, resulting in a smaller lithostatic stress in the downslope direction; the difference between the areas of both triangles is a measure of the gravitational driving stress responsible for glacier flow.

ated with gradients in these stresses (see, e.g., Van der Veen, 2013, Fig. 3.1 and Eqs. 3.8-3.9). Balance of forces is only meaningful if applied to flow-line segments, not single locations. Consequently, the concept of force balance at any location is inherently flawed. While many, if not most, glaciologists, Van der Veen (2013) included, often refer to driving stress or basal drag at location $x$, it would be more appropriate to refer to these quantities as areal averages. If the surface slope is calculated over a distance $2 \Delta x$, the associated driving stress is the average over the interval $(x-\Delta x, x+\Delta x)$, and similarly for basal drag. Nuancing common parlance to reflect this subtlety would render many discussions of glacier dynamics unnecessarily cumbersome and could be superfluous for most readers' understanding of the fundamentals of glacier dynamics.

\section{Discussion}

While the geometric force-balance approach is severely limited, it is worth exploring the central premise of Hughes' ideas, namely that the transition from sheet flow to shelf flow is achieved through basal buoyancy, with interior ice firmly grounded on bedrock and ice shelves floating in sea water. It should be noted that for both these end-member solutions, at any location the weight of an ice column is fully supported from directly below: terra firma in the case of grounded ice, and sea water for ice shelves. 
While not immediately obvious, the role of varying subglacial water pressure is included in the force-balance Eq. (2), namely though bridging effects (Van der Veen, 2013, Sect. 3.4). To clarify this, consider that resistive stresses are linked to strain rates, or velocity gradients, by invoking Glen's flow law for glacier ice (Van der Veen and Whillans, 1989; Van der Veen, 2013, Sect. 3.3):

$$
\begin{aligned}
& R_{x x}=B \dot{\varepsilon}_{\mathrm{e}}^{1 / n-1}\left(2 \dot{\varepsilon}_{x x}+\dot{\varepsilon}_{y y}\right)+R_{z z} \\
& R_{x y}=B \dot{\varepsilon}_{\mathrm{e}}^{1 / n-1} \dot{\varepsilon}_{x y} .
\end{aligned}
$$

Here, $B$ represents the temperature-dependent rate factor, and $n=3$ the flow-law exponent; $\dot{\varepsilon}_{\mathrm{e}}$ is the effective strain rate defined as the second invariant of the strain-rate tensor. The last term on the right-hand side of Eq. (17) is the vertical resistive stress defined as

$R_{z z}(z)=\sigma_{z z}+\rho g(h-z)$

The stress $R_{z z}$ represents the difference between the full vertical stress, $\sigma_{z z}$, and the lithostatic stress, or weight of the ice above some level. This term arises in Eq. (7) because Glenn's flow law relates strain rates to deviatoric stresses, rather than full stresses.

For brevity of notation, the along-flow resistive stress is written as the sum of a contribution associated with alongflow gradients in velocity (first term on the right-hand side of Eq. 17) and the vertical resistive stress:

$R_{x x}=R_{x x}^{(0)}+R_{z z}$

Force balance in the horizontal direction can then also be written as

$$
\begin{aligned}
& \tau_{d x}=\tau_{b x}-\frac{\partial}{\partial x}\left(H \widetilde{R}_{x x}^{(0)}\right)-\frac{\partial}{\partial y}\left(H \widetilde{R}_{x y}\right) \\
& -\frac{\partial}{\partial x} \int_{h-H}^{h} R_{z z}(z) d z .
\end{aligned}
$$

Where the weight of the ice is fully supported by the substrate below, the vertical resistive stress is zero. This is the assumption usually made when considering the budget of forces acting on glaciers (e.g., Van der Veen and Whillans, 1989). Locally, however, bridging effects may be important, for example where a water-filled cavity exists at the icebed interface (Van der Veen, 2013, Sect. 7.2). Where cavitation occurs and basal ice becomes separated from the bed, the cavity cannot support the weight of the ice leading to shear-stress gradients that effectively transfer the weight to surrounding areas where the ice is in contact with the bed, such that the areal average of the vertical resistive stress is zero. Thus, on a large scale, such as the length of ice streams and outlet glaciers, basal buoyancy is a non-issue where horizontal force balance is concerned. Indeed, Hughes (1998, Eq. 3.5) does not include bridging effects in his discussions and equates the total vertical stress at depth to the lithostatic stress.

Basal buoyancy may be important on ice streams and outlet glaciers according to the commonly adopted sliding relation in which sliding speed is inversely proportional to the effective basal pressure. Pfeffer (2007) suggests that this proportionality may explain rapid velocity increases on tidewater glaciers and Greenland outlet glaciers: as these glaciers thinned and thickness approached flotation, the effective basal pressure approached zero, resulting in a large increase in sliding velocity. Another possibility is that increased basal buoyancy reduces basal drag, thereby allowing glaciers to move faster. The importance of these effects can be evaluated from analysis of time series of surface speed and glacier geometry, or using numerical models based on the balance Eq. (7).

The primary difference between shelf flow and stream flow is that ice shelves are floating in water and basal drag is zero, whereas for sheet flow, basal drag provides most resistance to flow. Thus, it would seem reasonable to propose that the transition from sheet to shelf flow involves a gradual reduction in basal resistance, perhaps associated with the presence within deforming sediments, or gradual drowning of bed obstacles. As basal drag becomes less important, longitudinal stress gradients and lateral drag must increase and provide most or all resistance to the flow of ice streams.

\section{Concluding remarks}

The geometric approach to ice sheet modeling links ice-bed coupling directly to the stresses that resist horizontal gravitational motion (Hughes, 2008, p. 34). This basal buoyancy supposedly translates into a major component of gravitational forcing by which ice sheets discharge ice into the sea (Hughes, 2003). As shown in this contribution, the geometric force balance as presented by Hughes in a series of publications cannot be successfully applied to ice streams and outlet glaciers. This is not to say that a geometric approach is inherently flawed - if implemented correctly it should produce consistent and correct results, but this has yet to be achieved.

The claim that the analytic force-budget approach fails to account for basal buoyancy and excludes a "water buttressing force" on ice streams is incorrect. Equation (7) describing the depth-integrated balance of horizontal forces is derived without making any simplifying assumptions and applies equally well to floating ice shelves and firmly grounded interior ice. If some force is missing from this equation, this force must also be missing from the momentum balance equations that form the starting point for deriving Eq. (7).

Hughes is correct that ice streams and outlet glaciers represent the transition from sheet flow and shelf flow and that much remains to be understood about the nature of this transition. Advantageously, ongoing rapid changes on many of the outlet glaciers have been well documented through 
time series of surface elevation and surface velocity. The latter, in particular, are powerful indicators of the distribution of stresses on glaciers because strain rates (velocity gradients) are directly linked to stresses through the flow law for glacier ice. Improved understanding of the dynamics of rapidly changing ice-sheet components will come from interpretation of strain rates and temporal changes therein.

Acknowledgements. I am indebted to Ken Jezek for his continued support and careful reading of this paper, and to Leigh Stearns for additional comments. This research was supported by NASA grant nos. FED0066542 and UNI0072622.

Edited by: F. Pattyn

\section{References}

Cuffey, K. M. and Paterson, W. S. B.: The Physics of Glaciers (Fourth Edition). Burlington MA: Butterworth-Heineman, 693 pp., 2010.

Engelder, T: Stress regimes in the lithosphere. Princeton NJ: Princeton University Press, 457 pp., 1993.

Hughes, T.: The Jakobshavn Effect, Geophys. Res. Lett., 13, 46-48, 1986.

Hughes, T.: On the pulling power of ice streams, J. Glaciol., 38, 125-151, 1992.

Hughes, T. J.: Ice Sheets, New York NY, Oxford University Press, 343 pp., 1998

Hughes, T. J.: Geometrical force balance in glaciology, J. Geophys. Res., 108, 2526, doi:10.1029/2003JB002557, 2003.

Hughes, T. J.: Holistic ice sheet modeling: a first-order approach, University of Maine, 188 pp., 2008.

Hughes, T.: Variations of ice bed coupling beneath and beyond ice streams: The force balance, J. Geophys. Res., 114, B01410, doi:10.1029/2008JB005714, 2009a.

Hughes, T.: Correction to "Variations of ice bed coupling beneath and beyond ice streams: The force balance," J Geophys Res 114, B04499, doi:10.1029/2009JB006426, 2009b.
Hughes, T.: Holistic Ice Sheet Modeling. A First-Order Approach. New York: Nova Publishers, 261 pp., 2012.

Hughes, T., Sargent, A., and Fastook, J.: Ice-bed coupling beneath and beyond ice streams: Byrd Glacier, Antarctica, J. Geophys. Res., 116, F03005, doi:10.1029/2010JF001896, 2011.

Hughes, T., Sargent, A., Fastook, J., Purdon, K., Li, J., Yan, J.-B., and Gogineni, S.: Sheet, stream, and shelf flow as progressive ice-bed uncoupling: Byrd Glacier, Antarctica and Jakobshavn Isbrae, Greenland, The Cryosphere, 10, 193-225, doi:10.5194/tc10-193-2016, 2016.

Pfeffer, W. T.: A simple mechanism for irreversible tidewater glacier retreat, J. Geophys. Res., 112, F03S25, doi:10.1029/2006JF000590, 2007.

Reusch, D. and Hughes, T.: Surface "waves" on Byrd Glacier, Antarctica, Antarct. Sci., 15, 547-555, 2003.

Turcotte, D. L. and Schubert, G.: Geodynamics (Second Edition), Cambridge, Cambridge University Press, 456 pp., 2002.

Van der Veen, C. J.: Fundamentals of Glacier Dynamics, Boca Raton, Taylor \& Francis, 389 pp., 2013.

Van der Veen, C. J. and Whillans, I. M.: Force budget: 1. Theory and numerical methods, J. Glaciol., 35, 53-60, 1989.

Van der Veen, C. J. and Payne, A. J.: Modelling land-ice dynamics. In: Mass balance of the cryosphere: observations and modelling of contemporary and future changes, edited by: Bamber, J. L. and Payne, A. J., Cambridge, Cambridge University Press, 169-225, 2004.

Van der Veen, C. J., Stearns, L. A., Johnson, J., and Csatho, B.: Flow dynamics of Byrd Glacier, East Antarctica, J. Glaciol., 60, 1053-1064, 2014.

Weertman, J.: Deformation of floating ice shelves, J. Glaciol., 3, 38-42, 1957.

Whillans, I. M.: Force budget of ice sheets. In: Dynamics of the West Antarctic Ice Sheets, edited by: Van der Veen, C. J. and Oerlemans, J., Dordrecht, Reidel, 17-36, 1987.

Whillans, I. M., Chen, Y. H., Van der Veen, C. J., and Hughes, T. J.: Force budget: III. Application to three-dimensional flow of Byrd Glacier, Antarctica, J. Glaciol., 119, 68-80, 1989. 